\title{
Etudes histopathologique et ultrastructurale de Thelobania contejeani (Microsporida, Nosematidae) parasite de l'Ecrevisse Austropotamobius pallipes Lereboullet (*)
}

\author{
par J. MAURAND et A. VEY \\ (Collaboration technique: J.-L. Duthoit et A. Amargier) \\ Laboratoire de Zoologie I ( $\mathrm{P}^{\mathrm{r}}$ O. TUZET), Université des Sciences \\ et Techniques du Languedoc, F 34000 Montpellier \\ et Station de Recherches cytopathologiques I.N.R.A.-C.N.R.S. \\ F 30380 Saint-Christol-les-Alès
}

\begin{abstract}
Résumé
Thelohania contejeani envahit principalement la musculature de l'écrevisse. La phase d'expansion végétative pendant laquelle elle se présente sous forme de schizontes et de plasmodes sporogoniaux est étroitement liée à la présence des myofibrilles et cesse avec la disparition de ces dernières. La formation de capsules dues à une réaction cellulaire de l'hôte a été observée.

L'étude ultrastructurale de $T$. contejeani montre que le golgi du sporoblaste sécrète, outre le filament polaire, des inclusions denses. La filament s'insère sur une large «coupelle » renversée Le pansporoblaste renferme entre les spores, des formations tubulaires à axe central monoliforme caractéristique.
\end{abstract}

(*) Ce travail a été effectué dans le cadre d'un Programme: Conseil Supérieur de la Pêche I.N.R.A., et avec la collaboration de l'Association des Fédérations de Pêche et de pisciculture de la Lozère. 


\section{Summary}

Thelohania contejeani chiefly invades the crayfish musculature. The extending vegetative phase is tightly linked with the presence of myofibrils and ceases when these disappear. We observed a capsule formation due to host-cells reactions.

The ultrastructural study of the microsporidia shows that the Golgi apparatus of the sporoblast secretes dense inclusions besides the polar filament. The filament attaches itself on a great upside down cup and the pansporoblast contains, between the spores, tubular formations with a monoliform core.

Les recherches que nous poursuivons concernant la pathologie des écrevisses, ont permis de mettre en évidence plusieurs maladies chez ces Crustacés. Ainsi, nous avons signalé dans deux articles préliminaires, la présence d'une microsporidie du genre Thelohania dans les écrevisses en Lozère (Vey, Vago, Charpy, 1972 ; Vey, Vago, 1972). On doit porter un grand intérêt à cet agent pathogène si l'on considère le grand danger qu'il représente pour les élevages de masse récemment entrepris.

Nous indiquerons ou rappellerons brièvement quelques données morphologiques concernant cette Thelohania nous permettant de l'identifier à l'espèce $T$. contejeani Henneguy. Mais surtout, nous nous proposons, dans la présente note, de préciser d'abord la progression de la microsporidiose dans le tissu musculaire, qui est le tissu le plus largement infecté, ensuite les principaux traits ultrastructuraux de l'agent pathogène. Ces derniers détails permettront de distinguer au fur et à mesure que leur ultrastructure sera connue $T$. contejeani des nombreuses autres espèces de Thelohania parasites de Crustacés Décapodes. En effet, les longueurs sporales de ces espèces sont toutes comprises entre 3 et $5 \mu$ et ce critère taille peut apparaître insuffisant.

Nos observations ont été effectuées sur des écrevisses de l'espèce Austropotamobius pallipes Lereboullet, 1958. Après leur capture, ces animaux sont maintenus au laboratoire en cellule climatisée à $17^{\circ}$ et placés dans des bacs de réserve, les lots d'origines différentes étant conservés séparément. La fréquence des animaux spontanément infectés atteint parfois $10 \%$ des animaux examinés.

\section{L'agent pathogène.}

Cette microsporidie possède des spores très légèrement piriformes, groupées par huit, mesurant sur frottis frais 3,5 à $4 \mu$ de long pour une largeur maximum de $2 \mu$. Henneguy et Thélohan (1892) donnent une longueur comprise entre 2 et $3 \mu$, mais ils précisent que leurs mesures sont effectuées sur du matériel fixé. Ceci justifie cette différence de longueur avec nos mesures faites sur frottis frais, la spore subissant une rétraction lors de la fixation. Il n'y a ainsi pas d'obstacle à ce que les deux espèces considérées soient identiques. La dévagination du filament polaire s'obtient facilement, soit en soumettant les spores à des alternances de froid et de chaud 
accompagnées d'une pression sur la lamelle, soit par la méthode de Kramer (1960): dessiccation suivie de réhydratation. Sa longueur moyenne est comprise entre 20 et $30 \mu$, mais elle peut atteindre $50 \mu$.

\section{La microsporidie dans les tissus.}

L'examen au microssope d'un fragment de muscle dilacéré est nécessaire pour établir un diagnostic au début de la maladie, alors que l'aspect macroscopique de l'écrevisse permet très difficilement de deviner la présence d'une telle infection. Par contre, en fin d'évolution, l'abdomen de l'écrevisse devient blanc d'où les noms évocateurs donnés à la maladie: maladie de la queue blanche en Finlande (Sumari et Westman, 1969), maladie de la porcelaine en Allemagne (Shaperclaus, 1954).
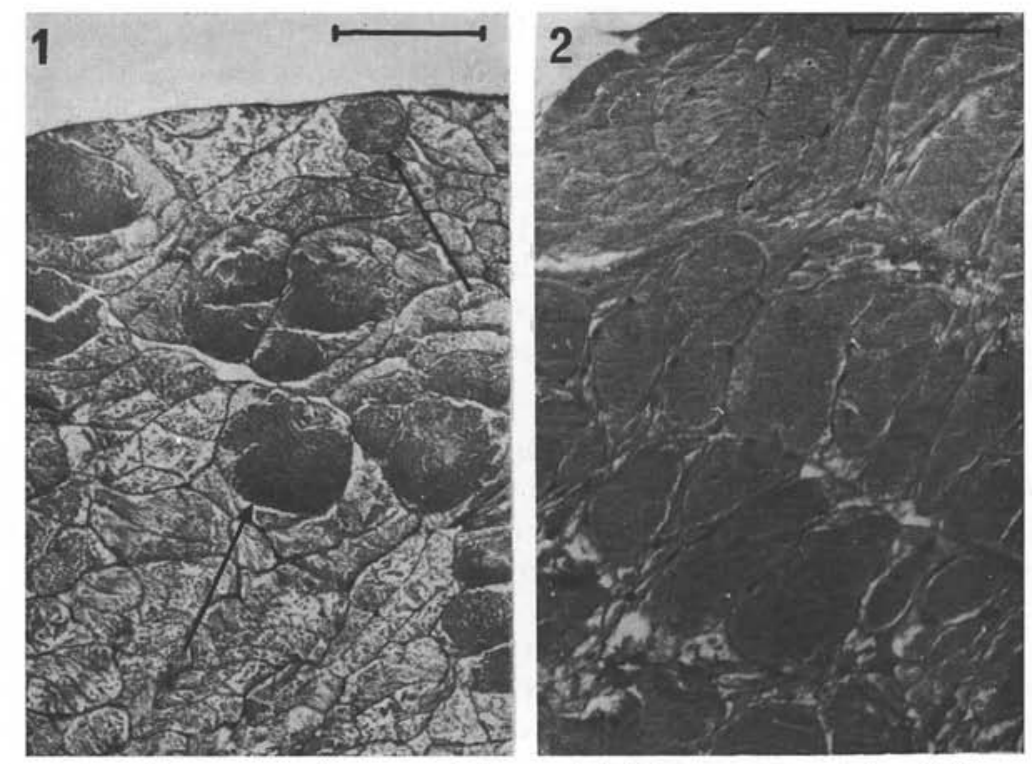

FIG. 1. - Envahissement du tissu musculaire par Thelohania contejeani. Les flèches soulignent deux foyers d'infection. (Fixation Carnoy-coloration azan de Heidenhain). Echelle $=50 \mu$

Fig. 2. - Invasion totale de la musculature avant la mort de l'Ecrevisse (Technique: comme 1). Echelle $=50 \mathrm{u}$

Sur coupes histologiques, nous avons décelé le parasite dans le tissu conjonctif entourant le tube digestif - mais non dans l'épithélium intestinal — dans le cerveau, la paroi du vaisseau dorsal et la musculature qui est le lieu de prédilection pour le développement de la microsporidie. Nous avons suivi l'évolution du processus pathologique dans les muscles abdominaux en comparant des coupes histologiques de muscles sains à des coupes de muscles faiblement et fortement infectés.

Dans les muscles peu atteints (fig. 1), la microsporidie forme plusieurs foyers d'infection indépendants les uns des autres, chacun correspondant à une fibre qui 
est donc l'unité de base infectée. Chacun de ces foyers dissout les myofibrilles de la cellule musculaire dont le sarcolemme constitue provisoirement un sac pour la microsporidie. A ce stade, le parasite est en phase de dispersion végétative. Nous observons dans le champ de Cohnheim des schizontes fusiformes très fortement colorés par l'hématoxyline de Groat. La coloration à l'azan de Heidenhain montre les autres stades : plasmodes sporogoniaux jaunes, rosaces de la sporoblastogenèse et sporoblastes soulignés par de délicates traînées bleuâtres, spores rouges. Sporoblastogenèse et sporoblastes correspondent à la phase de sécrétion de la microsporidie mise en évidence par Maurand et Bouix (1970) chez Thelohania fibrata. Dans le cas de T. contejeani, il y a aussi une positivité avec le bleu alcian à $\mathrm{pH} 3,5$ indiquant la présence très probable de mucosubstances acides.

Lorsque les muscles sont fortement infectés, rompant le sarcolemme, le foyer infectieux déborde le cadre de la fibre et s'unit au foyer de la fibre voisine. Les foyers isolés se réunissent donc et leur extension cesse devant la barrière constituée par le conjonctif entourant un faisceau de fibres (fig. 2). Nous n'observons pas d'hypertrophies cellulaires, ni des accroissements de taille du noyau comme cela est fréquemment le cas dans les tissus infectés par des microsporidies chez d'autres Arthropodes (corps adipeux des Insectes). Il y a remplacement volume par volume du contenu de la fibre par les stades de la microsporidie. On n'observe plus alors de schizontes et de plasmodes sporogoniaux dans les coupes, les sporoblastes et les spores étant les seuls stades présents. La positivité au bleu alcian disparaît avec la fin de la sporoblastogenèse.

Il nous paraît intéressant de mentionner ici l'existence de phénomènes d'encapsulement (fig. 3) observés à la limite du tissu musculaire. Des cellules-hôtes renfermant la microsporidie sont entourées par une capsule comprenant au moins cinq assises cellulaires. Quelques cellules de ces dernières renferment de grosses inclusions denses, qui les font identifier aux hémocytes à granulations osmiophiles de grande taille, dont la participation aux réactions vis-à-vis des champignons a récemment été mise en évidence chez l'écrevisse (Unestam et Nylund, 1972). Dans la cellule parasitée et encapsulée, des phénomènes de dégénérescence sont en cours, avec de nombreux systèmes membranaires présentant l'aspect de corps «myéliniques » ou « résiduels » qui constituent l'étape ultime de l'évolution des formations à activité lytique. L'encapsulement de microsporidies par des cellules est relativement rare et son rôle dans la défense de l'organisme probablement réduit. Telle est l'opinion de Brooks (1971) qui décrivit le premier cas vrai de réaction cellulaire intense chez Manduca sexta infectée par Nosema sphingidis.

De cette étude histopathologique, nous retenons particulièrement deux faits :

- la diminution de l'activité musculaire de l'écrevisse parasitée, constatée au myographe par Henneguy et Thelohan s'explique par la destruction parasitaire des myofibrilles et de certaines membranes cellulaires, sièges, soit de la contraction ellemême, soit de phénomènes électriques lors de la contraction, 
- le parallélisme entre le développement de la microsporidie et l'état du tissu envahi montre une relation entre les myofibrilles et les schizontes. Tout se passe comme si les schizontes se multiplient tant qu'il y a des myofibrilles à « dissoudre » Quand toutes les myofibrilles d'une cellule sont disloquées et que les schizontes ne peuvent franchir la barrière de conjonctif interfasciculaire, ces derniers se transforment intégralement en sporontes.

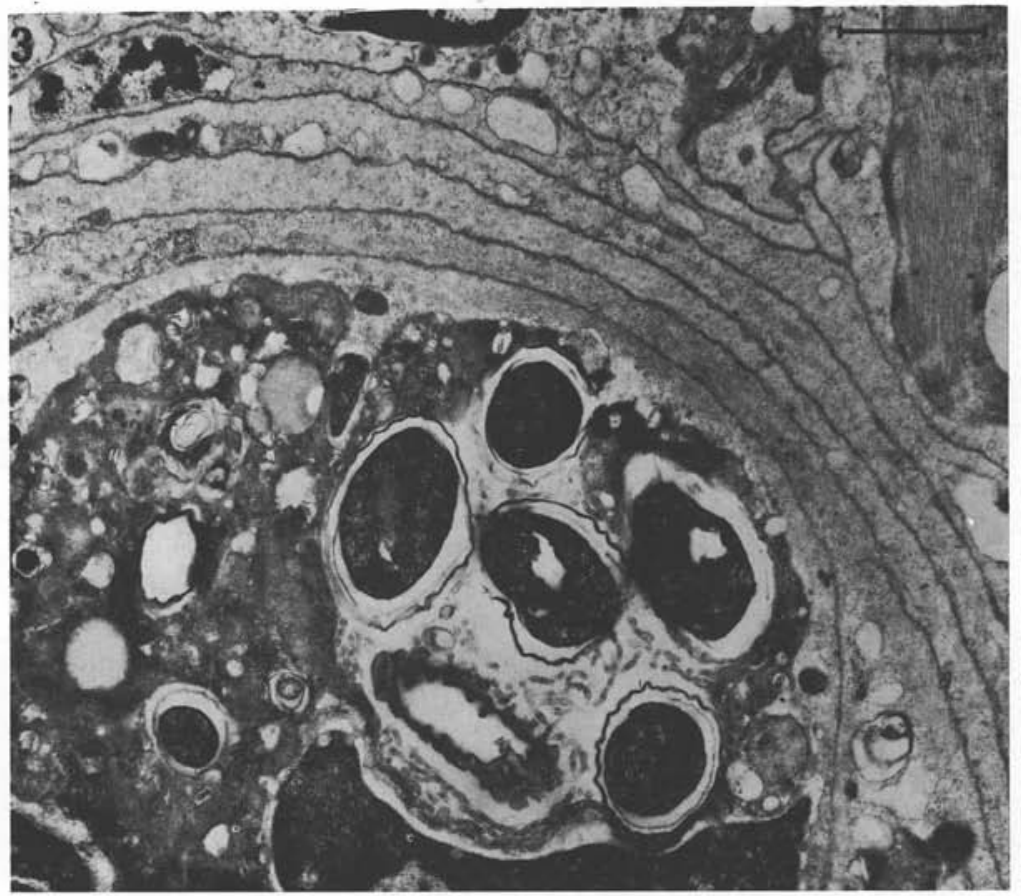

FIG. 3. - Encapsulement de pansporoblastes de $T$. contejeani. (Colorations acétate d'uranyle et citrate de plomb. Micr. électr.). Echelle $=1,4 \mu$

\section{Etude ultrastructurale de $T$. contejeani.}

L'étude de la structure fine de Thelohania contejeani a été réalisée sur des fragments de muscles infectés, fixés par le glutaraldéhyde à $2 \%$ dans un tampon cacodylate à $\mathrm{pH} 7,2$, post-fixés dans l'acide osmique et inclus dans l'épon. Les coupes ultrafines, contrastées par l'acétate d'uranyle en solution alcoolique et le citrate de plomb ont été examinées au microscope Hitachi Hu 11 C.S. Nos observations portent sur les schizontes, les sporoblastes, les spores ainsi que les autres éléments du contenu de la membrane pansporoblastique.

Les schizontes (fig. 4) sont caractérisés par la possession d'un ou plusieurs systèmes nucléaires dits diplocaryons autour desquels se disposent les cisternes apla. 

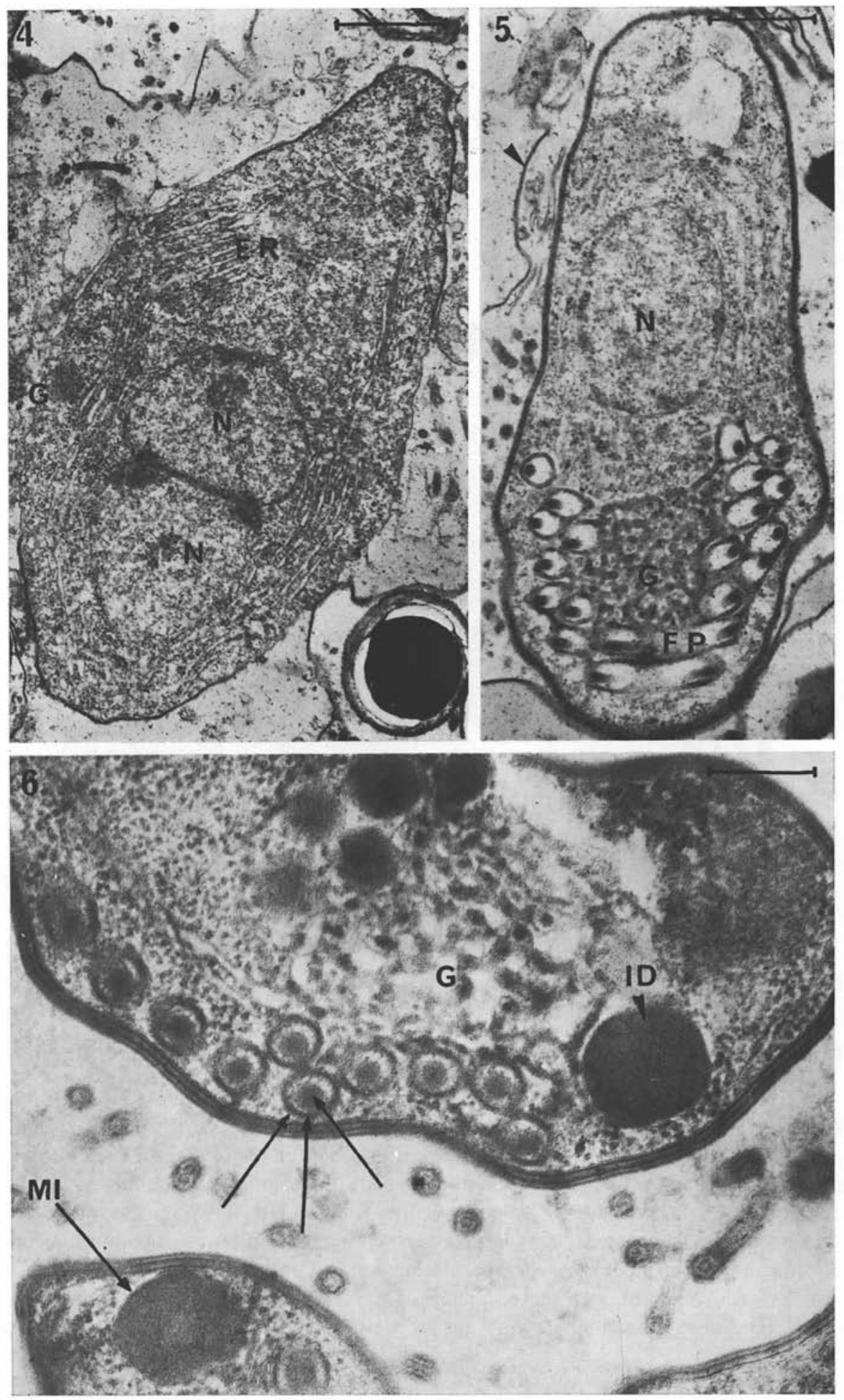
ties de l'ergastoplasme. Nous distinguons çà et là, l'organite formé par rapprochement de vésicules denses aux électrons qui serait un appareil de Golgi primitif (Vavra, 1965). Des restes de myofibrilles jouxtent généralement les schizontes.

Les sporoblastes (fig. 5), uninucléés, se forment au nombre de huit à partir d'un plasmode sporogonial. Dès leur individualisation, ils élaborent le filament polaire avant que nous discernions la première ébauche du polaroplaste. Dans 1a partie postérieure du sporoblaste, le golgi se dilate, ses vésicules pouvant s'étirer en de courts tubules (fig. 5 et 6), Il rappelle indiscutablement le golgi en cours d'élaboration du filament polaire dans les sporoblastes de Nosema vivieri (Vinckier et coll., 1971), de Pleistophora sp. (Szollosi, 1971), Stempellia simulii (Maurand et Manier, 1968). Ses vésicules sont étroitement associées aux spires naissantes du filament polaire. Outre ce rôle morphogénétique, le golgi, dans certaines de ses vésicules, concentre ou synthétise un matériel dense aux électrons prenant la forme de sphérules entourées d'une membrane (fig. 6). Les plus grosses atteignent $0,2 \mu$ de diamètre, les petites sont denses à partir de $0,1 \mu$. Ces inclusions ne sont pas rares chez les microsporidies. Elles sont signalées dans les sporoblastes ou spores de Coccospora gatesi (De Puytorac et Tourret, 1963), Nosema sp. (Sprague et coll., 1968). Présentes ou absentes selon les espèces de microsporidies considérées, elles peuvent donc servir à les caractériser.

Nous décrivons chez la spore mûre, l'enveloppe, le filament polaire, le polaroplaste, le sporoplasme uninucléé.

L'enveloppe sporale est formée de trois parties qui sont de l'extérieur vers l'intérieur de la spore: une exospore bimembranaire issue de l'enveloppe sporoblastique, une endospore transparente aux électrons, une fine membrane ectoplasmique (fig. 7).

Le filament polaire est étroitement associé dans sa région antérieure à une formation en coupelle renversée (ou bien en ancre si on considère la seule section de l'organite: Stanier et coll., 1968) (fig. 7). Cette structure basale diffère sensiblement de celle que nous avons observée chez Plistophora simulii (Maurand, 1966) ainsi que de celle décrite par Lom et Corliss (1967) chez Plistophora hyphessobryconis. Le filament s'enroule en formant dix tours de spire dans la région postérieure de la spore. Son diamètre est d'environ $800 \AA$ dans sa partie postérieure et l'aspect de la section transversale varie durant la maturation du sporoblaste. Le filament est entouré d'une membrane et chez le sporoblaste ou la jeune spore, il comprend une assise externe dense aux électrons et une partie interne transparente, renfermant un axe sombre (fig. 6). Ceci correspond de très près aux images données par Vinckier et

FIG. 4. - Schizonte à diplocaryon (N.N.). Noter la présence d'un ergastoplasme (ER) et d'un golgi (G). (Micr. électr.). Echelle $=1 \mu$

FIG. 5. - Sporoblaste âgé. On observe le noyau sphérique (N), le filament polaire (FP) étroitement associé au golgi (G). La flèche indique la membrane pansporoblastique. (Micr. électr.). Echelle $=0,8 \mu$

FIG. 6. - Détail d'un sporoblaste montrant la structure du jeune filament polaire (3 flèches) et les inclusions denses (ID) entourées d'une membrane (MI). Les vésicules golgiennes sont plus ou moins étirées en tubules. (Micr. électr.). Echelle $=0,7 \mu$ 
coll. (1971) pour Nosema vivieri. A la maturité de la spore, l'axe sombre est subdivisé en un fin anneau concentrique à l'assise externe dense et un nouvel axe sombre.

Le polaroplaste, limité antérieurement par la cupule basale du filament polaire, a une structure lamellaire et montre une alternance de zones claires et sombres. Mais

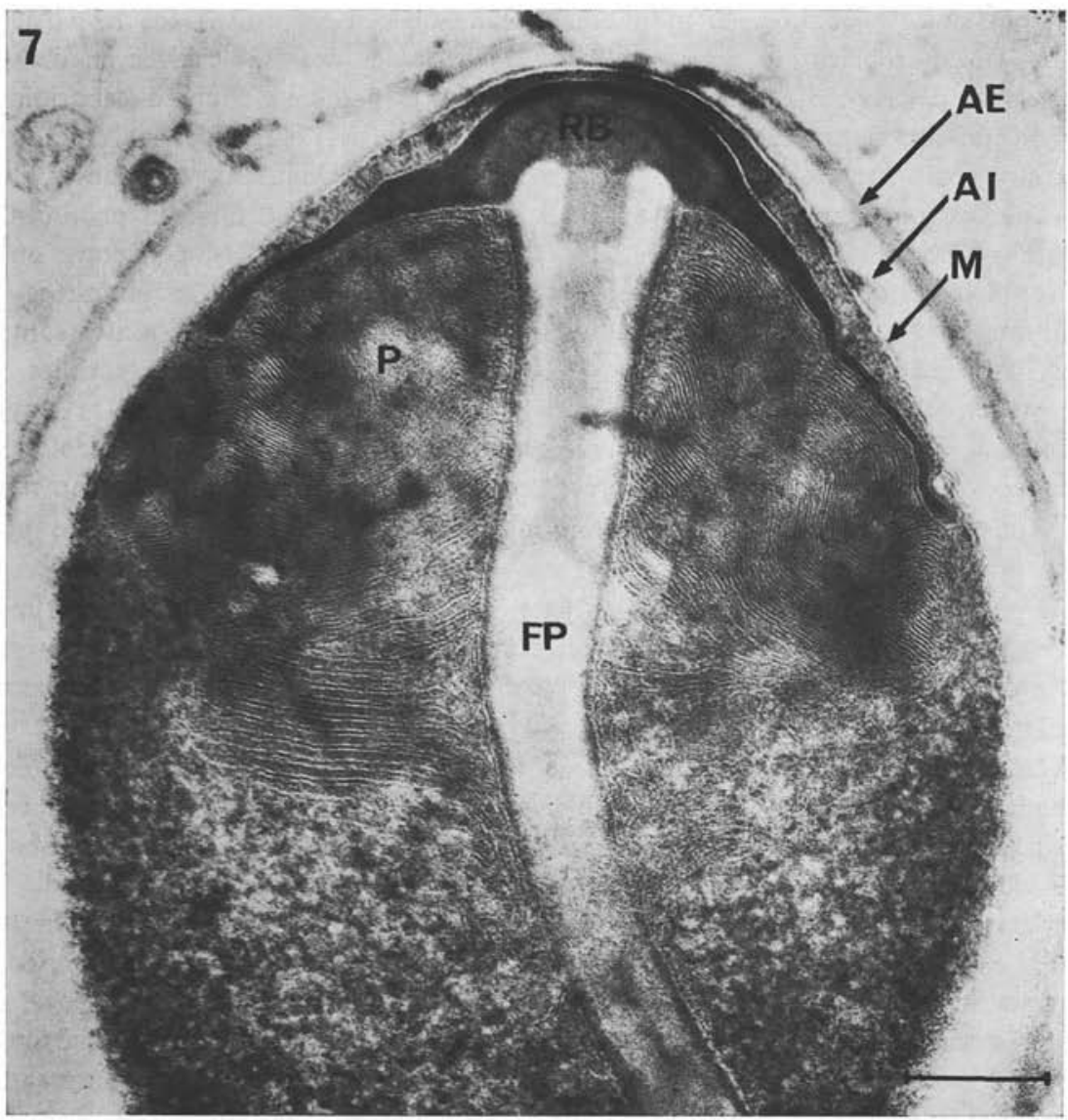

Fig. 7. - Détail de la région antérieure de la spore. L'enveloppe sporale est composée de trois éléments: exospore (AE), endospore (AI) et membrane plasmique $(\mathrm{M})$. Le filament s'insère sur un organite très élaboré pour lequel nous choisirons provisoirement la dénomination de renforcement basal $(\mathrm{RB}) . \mathrm{P}=$ polaroplaste (Micr. électr.). Echelle $=1,4 \mu$

si dans la région antérieure ces deux zones ont sensiblement la même épaisseur, dans la région postérieure du polaroplaste, les bandes sombres ont deux épaisseurs se succédant régulièrement (fig. 7).

Le noyau de la spore est unique. Régulièrement sphérique chez le sporoblaste, il s'étire, s'étrangle dans la région moyenne et se replie sur lui-même dans la spore mûre entre les tours de spire du filament et la partie postérieure du polaroplaste. Ceci 
explique que, sur certaines coupes, les spores semblent renfermer deux noyaux très voisins correspondant, en fait, aux deux parties du noyau recourbé. Mais nous savons déjà que les formes non globuleuses existent chez les noyaux de spores mûres de microsporidies: noyau en bissac de la spore de Metchnikovella hovassei (Vivier 1965), noyau contourné de Plistophora simulii (Maurand, 1966). Le noyau du sporoblaste subit donc d'importantes modifications morphologiques pendant la maturation de la spore.

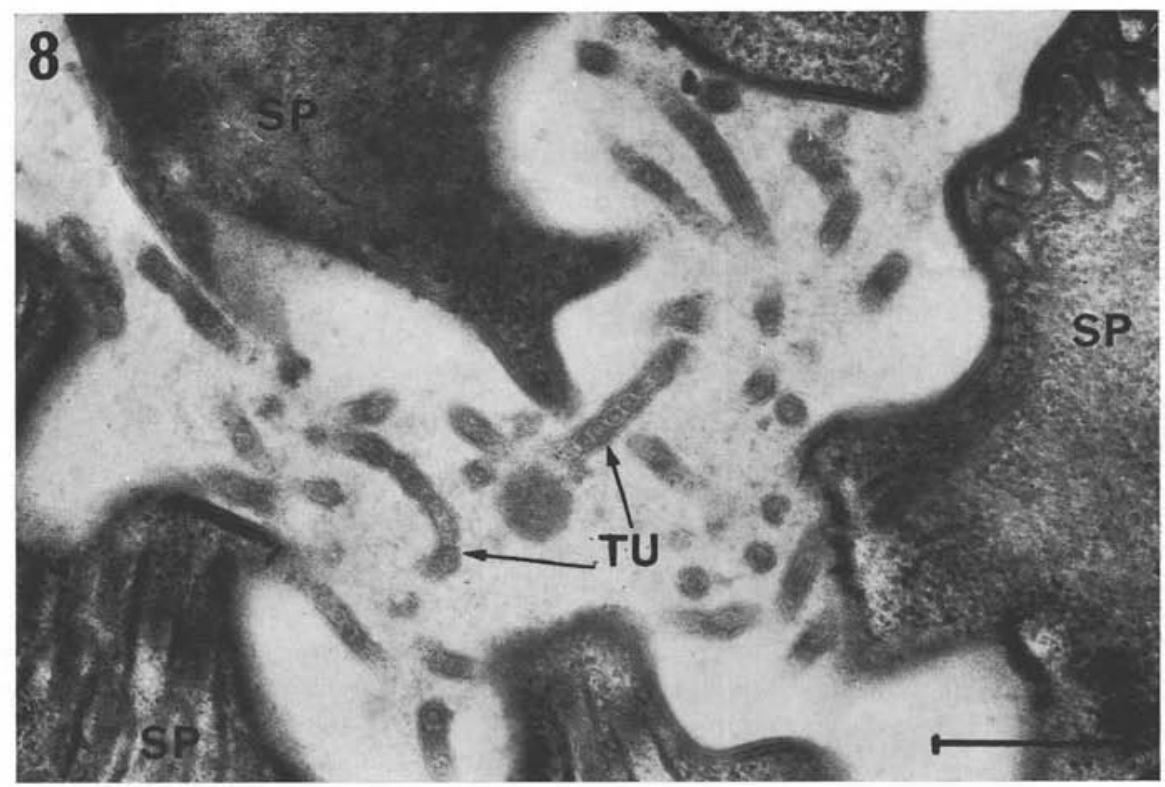

FIG. 8. - Formations tubulaires (TU) avec axe monoliforme entre les jeunes spores (SP). (Micr. électr.). Echelle $=0,4 \mu$

La membrane pansporoblastique renferme, outre les spores, d'abord de fines granulations denses aux électrons groupées en amas, ensuite des formations flexueuses structurées. La longueur de ces dernières est difficile à déterminer et nous ne savons pas si un pansporoblaste renferme un ou plusieurs filaments. Leur diamètre atteint $600 \AA$ et ils renferment un axe monoliforme, ce qui explique les aspects observés en coupes longitudinales et tangentielles (fig. 8). Il s'agit donc de formations extrêmement originales.

Les granulations du pansporoblaste, denses aux électrons, sont observées pour la première fois par Vavra (1965) chez diverses espèces dont Thelohania bracteata. Chez Telomyxa glugeiformis, elles accolent les deux spores contenues dans le pansporoblaste (Codreanu et Vavra, 1970). Des formations filamenteuses sont appendues à la paroi sporale de Plistophora debaisieuxi (Vavra, 1965), à la membrane pansporoblastique de Stempellia simulii (Maurand et Manier, 1968), s'échappent du 
matériel granulaire de la diplospore de Telomyxa glugeiformis (Codreanu et Vavra, 1970), occupent une partie de l'espace compris entre l'enveloppe sporale et la membrane pansporoblastique de Tuzetia infirma (Maurand et coll., 1971 ; section transversale des filaments fig. 1 , p. s.). Chez cette dernière espèce et chez $T$. contejeani, les filaments sont précédés par des productions granuleuses qui disparaissent pendant la sporogenèse, le pansporoblaste mûr ne renfermant que les filaments. Existet-il une relation autre que celle de leur succession dans le temps entre les granules et les filaments observés? Il n'est pas possible de répondre à cette question dans l'état actuel de nos connaissances, mais il nous paraît nécessaire de distinguer, au moins à titre provisoire, d'une part, les granulations denses aux électrons, d'autre part, les formations filamenteuses structurées.

Nous pouvons résumer ainsi les caractéristiques ultrastructurales de Thelohania contejeani : spore à noyau unique replié, filament polaire s'insérant sur une large coupelle renversée, inclusions denses sécrétées par le golgi dans la partie postérieure de la spore, présence entre les spores mûres de formations filamenteuses pourvues d'un axe monoliforme.

\section{Bibliographie}

Brooks (W. M.), 1971. - The inflammatory re:ponse of the tobacco Hornworm Manduca sexta, to infection by the microsporidian Nosema sphingidis. J. Invertebr. Pathol., 17, 87-93.

Codreanu (R.) et VAVRa (J.), 1970. - The structure and ultrastructure of the microsporidian Telomyxa glugeiformis Leger and Hesse, 1910, parasite of Ephemera danica (Müll.). nymphs. J. Protozool., 17, 374-384.

Henneguy (G.) et Thelohan (P.), 1892. - Myxosporidies parasites des muscles chez quelques Crustacés Décapodes. Ann. Micrographie, 4, 617-641.

Kramer (J.-P.), 1960. - Observations on the emergence of the microsporidian sporoplasm. J. Insect Pathol., 2, 433-439.

LOM (J.) et CoRLISs (J. O.), 1967. - Ultrastructural observations on the development of the microsporidian protozoon Plistophora hyphessobryconis Shaperclaus. J. Protozool., 14, 141-152.

Maurand (J.), 1966. - Plistophora simulii (Lutz et Splendore, 1904), microsporidie parasite des larves de Simulies: cycle, ultrastructure, ses rapports avec Thelohania bracteata (Strickland, 1913). Bull. Soc. Zool., Fr., 91, 621-629.

— et Bourx (G.), 1969. - Mise en évidence d'un phénomène sécrétoire dans le cycle de Thelohania fibrata (Strickland, 1913), microsporidie parasite des larves de Simulium. C.R. Acad. Sci., 269, 2216-2218.

—, Fize (A.), Fenwick (B.) et Michel (R.), 1971. - Etude au microscope électronique de Nosema infirmum Kudo, 1921, microsporidie parasite d'un Copépode Cyclopoïde ; création du genre nouveau Tuzetia à propos de cette espèce. Protistologica, 7, 221-225. 
— et Manier (J.-F.), 1968. - Une microsporidie nouvelle pour les larves de Simulies. Protistologica, 3, 445-449.

Puytorac (P. de) et Tourret (M.), 1963. - Etude de kystes d'origine parasitaire (microsporidies ou grégarines) sur la paroi interne du corps des vers Megascolecidae. Ann. Parasit. Hum. Comp., 38, 861-874.

Shaperclaus (W.), 1957. - Fischkrankheiten. Akademie Verlag, Berlin, 426-435.

Sprague (V.), Vernick (S. H.) et Lloyd (B. J. Jr.), 1968. - The fine structure of Nosema sp. Sprague, 1965 (Microsporida, Nosematidae) with particular reference to stages in sporogony. J. Invertebr. Pathol., 12, 105-117.

Stanier (J. E.), Woodhouse (M. A.) et Griffin (R. L.), 1968. - The fine structure of the spore of Nosema spelotremae, a microsporidian parasite of Spelotrema metacercaria encysted in the Crab Carcinus maenas. J. Invertebr. Pathol., 12, 73-82.

Sumari (O.) et Westman (K.), 1969. - The crayfish parasite Thelohania contejeani Henneguy (Sporozoa, Microsporidia) found in Finland. Ann. Zool. Fen., 7, 193-194.

Szollosi (D.), 1971. - Development of Pleistophora sp. (microsporidian) in eggs of the Polychaete Armandia brevis. J. Invertebr. Pathol., 18, 1-15.

Unestam (T.) et Nylund (J. E.), 1972. - Bool reactions in vitro in Crayfish against a fungal parasite. Aphanomyces astaci. J. Invertebr. Pathol., 19, 94-106.

VAVRA (J.), 1965. - Etude au microscope électronique de la morphologie et du développement de quelques microsporidies. C.R. Acad. Sci., 216, 3467-3470.

VeY (A.) et VAGo (C.), 1972. - Recherches sur les maladies actuelles des Ecrevisses de France. Ann. Hydrobiol. I.N.R.A., (sous presse).

—, - et Charpy (R.), 1971. - Une microsporidiose à Thelohania de l'Ecrevisse Austropotamobius (Atlantoastacus) pallipes en France. C.R. Acad. Agric. France, 57, 1540-1543.

Vinckier (D.), Devauchelle (G.) et Prensier (G.), 1971. - Etude ultrastructurale du développement de la microsporidie Nosema vivieri V. D. et P., 1970. Protistologica, 7, 273-287.

VIVIER (E.), 1965. - Etude au microscope électronique de la spore de Metchnikovella hovasei; appartenance des Metchnikovellidae aux microsporidies. C.R. Acad. Sci., 260, 6982-6984. 